\title{
Time to get personal with fluid administration and keep contrast-induced injury at bay
}

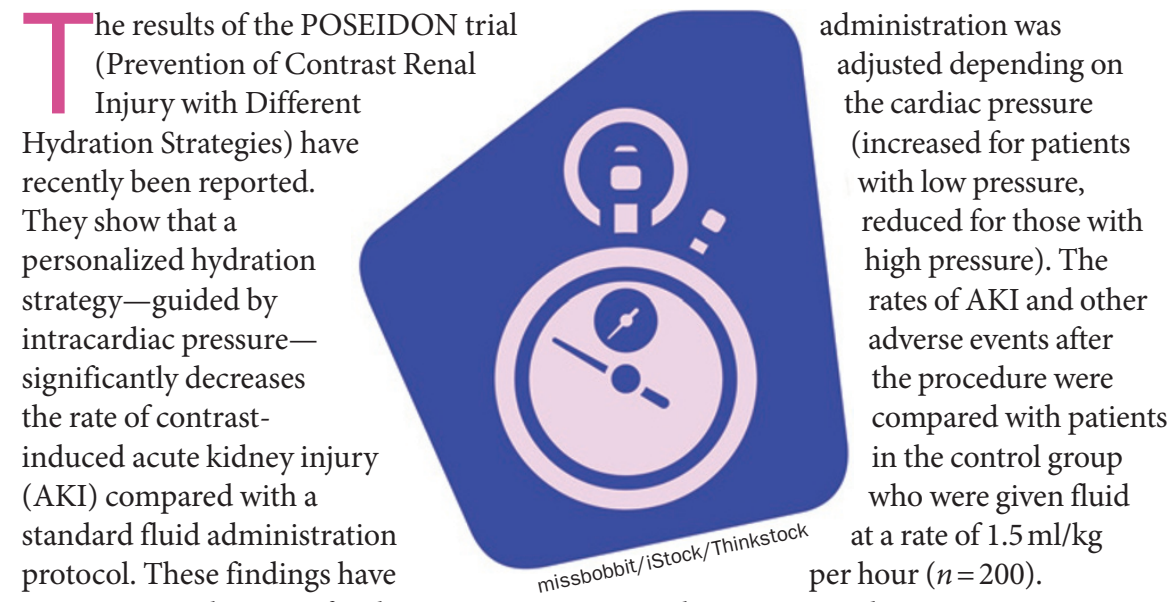
important implications for the considerable number of patients who are at risk of contrast-induced AKI.

In patients who are undergoing cardiac catheterization, current clinical practice is to administer fluid around the time of exposure to the imaging agent to avoid contrast-induced AKI. "While fluid administration is generally recommended, preferably with normal saline, there are no head to head comparisons of normal saline fluid administration protocols in patients at risk of contrast nephropathy," explains lead investigator Somjot Brar. Furthermore, the optimal volume, rate and duration of fluid administration has not been determined in a controlled study, leading to considerable variation in practice. "The lack of clinical trial data on how best to administer normal saline for the prevention of contrast nephropathy is surprising."

In their study, the researchers used left ventricular end-diastolic pressure, a known intravascular volume metric, to guide volume expansion in patients undergoing cardiac catheterization $(n=196)$. The rate of fluid
The primary end point was an increase in serum creatinine concentration $>25 \%$ from baseline, or an absolute increase of $0.5 \mathrm{mg} / \mathrm{dl}$, within 4 days of the procedure. Secondary end points included all-cause mortality, myocardial infarction and the need for renal replacement therapy at 30 days and 6 months.

The overall incidence of contrastinduced AKI was $6.7 \%$ in the pressureguided group and $16.3 \%$ in the control group $(P=0.005)$. At 6 months, four patients in the control group and only one patient in the pressure-guided group required renal replacement therapy; this difference was not statistically significant. Furthermore, patients in the pressureguided group required more volume than those in the control group $(1,727 \mathrm{ml}$ versus $812 \mathrm{ml} ; P<0.0001)$. Patients who required larger volumes of saline - up to $5 \mathrm{ml} / \mathrm{kg}$ per hour in this study, the highest fluid administration rate studied to date-had lower rates of contrast-induced AKI than those who received smaller volumes. "I believe that linking the fluid administration rate to the left ventricular end-diastolic pressure is important to minimize the risk of volume overload," comments Brar.

Although promising, these results must be regarded carefully. Fluid administration, in particular at the aggressive rate studied in this trial, is not applicable to all patients undergoing cardiac catheterization. "Physicians should exercise caution in fluid administration for patients with volume overload, acute decompensated heart failure or severe valvular heart disease," explains Brar. "Further research is needed to identify preventative strategies in these patient populations for whom aggressive fluid administration might not be permissible."

Indeed, contrast-induced AKI is an area of active research, with researchers looking at a range of factors from biomarkers that can predict risk of injury before contrast administration to factors that might influence the severity of injury in different populations. Brar suggests that noninvasive methods that assess intravascular volume status might enable more-aggressive fluid administration in patients whose left ventricular enddiastolic pressure is not measured. More research is needed to identify what these noninvasive methods might be, and assess them in clinical trials.

"I hope that these data will renew the focus on the importance of fluid administration for the prevention of contrast nephropathy, and heighten the recognition that contrast nephropathy is associated with both short-term and long-term adverse events," concludes Brar.

Mina Razzak

\footnotetext{
Original article Brar, S. S. et al. Haemodynamic-guided fluid administration for the prevention of contrast-induced acute kidney injury: the POSEIDON randomised controlled trial. Lancet 383, 1814-1823 (2014).
} 